\title{
Surface Roughness of Nanofiller Composite Resin after Immersion in Black Tea
}

\author{
Tiffany Henry Phanestu ${ }^{1}$, Lasminda Syafiar ${ }^{2 *}$ \\ ${ }^{1}$ Faculty of Dentistry, University of Sumatera Utara, Medan, Indonesia \\ ${ }^{2}$ Department of Dental Material and Technology, Faculty of Dentistry, University of Sumatera \\ Utara, Medan, Indonesia \\ *Email: lasmindasyafiar@yahoo.com
}

\begin{abstract}
The objective of this study is to evaluate the surface roughness of a nanofiller composite resin after immersion in black tea for 1,2,3, and $4 \mathrm{~h}$. A total of 24 disk-shaped samples were fabricated from a nanofiller composite resin (Filtek Z350XT, 3M ESPE, USA) and divided into the following four test groups: group I for 1-h immersion, group II for 2-h immersion, group III for 3-h immersion, and group IV for 4-h immersion. All the specimens were suspended in distilled water at $37^{\circ} \mathrm{C}$ for $24 \mathrm{~h}$. The initial surface roughness (Ra) and the surface roughness after the immersion of the samples were measured using a profilometer (MarSurf M300, Germany). The differences in the surface roughness values were assessed using a paired t-test. The differences between the experimental groups were analyzed using one-way ANOVA; $p<0.05$ was accepted as statistically significant. Each test group showed a difference in surface roughness after the immersion in black tea, and significant differences were observed between the test groups $(p=0.00)$. A significant difference was observed in the surface roughness of the nanofiller composite resin after immersion in black tea for 1,2,3, and $4 \mathrm{~h}$.
\end{abstract}

Keywords: surface roughness, nanofiller composite resin, black tea

\section{Introduction}

One of the most popular tooth restoration materials is the composite resin, a toothcolored restoration material that is widely used because of its good esthetic and physical characteristics and a great bonding system. Unfortunately, similar to other restoration materials, composite resin also has several limitations, such as a gap formation after polymerization that would cause marginal discoloration and leakage. Some developments have been made for improving the mechanical characteristics of the composite resin, such as the smaller size of the filler, a better bonding system, curing refinement, and the sealing system. The newest type of composite resin is the nanofiller, which is formulated by a nanocluster filler and nanomers. A combination of nanomer-sized particles and nanocluster formulation can decrease the interstitial gap of the filler and can thus increase the load of the filler, result in better physical characteristics and polishing retention, and decrease the surface roughness [1].

Black tea is one of the most consumed drinks in Indonesia. Among the three types of tea, viz., black tea, green tea, and oolong tea, black tea is the most popular one because of its greater taste and several advantages to the body [2]. 
One of the surface characteristics of the composite resin, especially roughness, is highly influenced by an acidic environment, such as the acid that is present in drinks [3].

Previous studies have shown that under an acidic condition, the restoration material degrades over time causing a difference in the surface roughness. Cresus et al. have shown that the immersion of composite resin in coffee, soft drinks, and coffee mix with soft drink can decrease the translucency and increase the surface roughness of the composite resin [3].

A restoration material with a smooth surface provides the maximum esthetic effect and durability. Surface roughness can lead to several clinical problems such as staining, plaque accumulation, recurrent caries, and periodontal disease. A smooth surface of the composite resin can decrease the possibility of forming a deposit of plaque and calculus and decrease the superficial discoloration of the composite resin [4]. The aim of this study was to evaluate the surface roughness of a nanofiller composite resin after immersion in black tea for $1,2,3$, and $4 \mathrm{~h}$.

\section{Methods}

\subsection{Preparation of the composite resin}

A total of 24 disk specimens from a nanofiller composite resin (Filtek Z350XT, 3M ESPE, USA) were prepared using a steel mold with the dimensions of 8-mm diameter and 2- mm thickness. The materials were condensed into the mold using a plastic instrument. The molds were overfilled and a cellophane strip was used to cover the surface, and two objective glasses were placed at the bottom and upper part of the mastercast to achieve the same thickness. The samples were cured using a lightcuring unit for $20 \mathrm{~s}$ with a gap of $1 \mathrm{~mm}$ from the sample surface in an upright position. Curing was performed from under and above the sample. After setting, the sample was taken out from the mold. Excessive samples were polished using a bur or a sandpaper. The bottom part of the samples was marked. The 24 specimens were divided into the following four test groups: group I for 1-h immersion, group II for 2-h immersion, group III for 3-h immersion, and group IV for 4-h immersion.

\subsection{Measurement of surface roughness}

The surface roughness was measured using a profilometer (MarSurf M300, Germany). To measure the roughness value, the stylus was placed at the measurement point and moved across the surface horizontally along a straight line as far as $8 \mathrm{~mm}$. This procedure was repeated three times for each specimen, and the average value was considered to be the Ra value. The surface roughness was measured before and after the application of topical fluoride gel.

\subsection{Immersion in black tea}

Black tea solution was prepared by pouring $120 \mathrm{~g}$ of tea into $1200 \mathrm{~mL}$ of hot water at a temperature of $100^{\circ} \mathrm{C}$. This solution was left undisturbed for $5 \mathrm{~min}$ and then filtered to obtain same particles. Four different containers were labeled according to the groups, and $300 \mathrm{~mL}$ of black tea solution was poured into each container. All 
the containers were placed in a water bath at $37^{\circ} \mathrm{C}$. When the temperature of the solutions reached $37^{\circ} \mathrm{C}$, the composite resin samples that were numbered were immersed in each container. Every container contained six samples. All pots were then placed in the water bath as follows: group I for 1-h immersion, group II for 2-h immersion, group III for 3-h immersion, and group IV for 4-h immersion. The samples were then taken out and dried using tissue paper. Each sample of each group was then taken out for surface roughness measurements.

\section{Result}

An increase in the surface roughness of the composite resin was observed after the immersion in black tea for $1,2,3$, and $4 \mathrm{~h}$ as shown by the paired t-test.

Table 1. The mean value of the composite resin surface roughness after immersion in black tea for $1,2,3$, and $4 \mathrm{~h}$.

\begin{tabular}{|c|c|c|c|}
\hline Test Group & $\begin{array}{c}\text { Initial Surface } \\
\text { Roughness }(\boldsymbol{\mu m})\end{array}$ & $\begin{array}{c}\text { Final Surface } \\
\text { Roughness }(\boldsymbol{\mu} \mathbf{m})\end{array}$ & $\begin{array}{c}\text { Changes in Surface } \\
\text { Roughness }(\boldsymbol{m} \mathbf{m})\end{array}$ \\
\hline Group 1 & $0,24 \pm 0,02$ & $0,24 \pm 0,02$ & 0,001 \\
\hline Group 2 & $0,24 \pm 0,02$ & $0,25 \pm 0,02$ & 0,005 \\
\hline Group 3 & $0,24 \pm 0,01$ & $0,25 \pm 0,01$ & 0,007 \\
\hline Group 4 & $0,22 \pm 0,02$ & $0,24 \pm 0,02$ & 0,01 \\
\hline
\end{tabular}

As shown in Table 1. group IV exhibited the greatest changes in surface roughness $(0.01233 \mu \mathrm{m})$ compared to those in group I $(0.00183 \mu \mathrm{m})$.

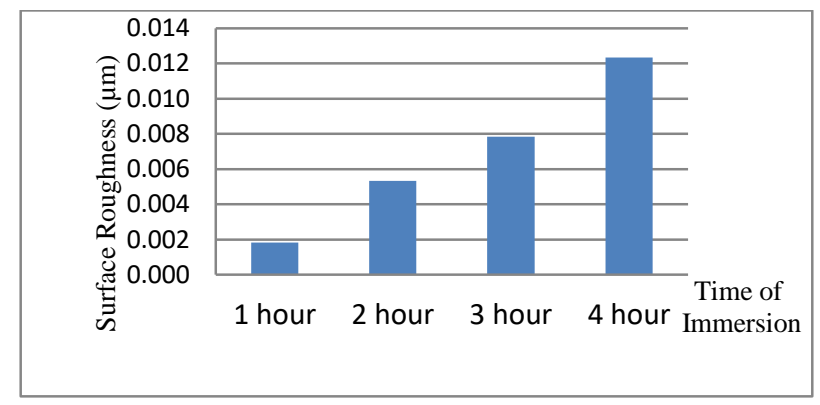

Fig. 1 Graph of the changes in the surface roughness.

The paired t-test analysis revealed significant differences in the surface roughness before and after immersion in black tea in both the treatment groups $(p=0.00)$. One-way ANOVA also showed significant differences in the surface roughness of the composite resin nanofiller in each immersion group $(p=0.00)$.

\section{Discussion}

The results of this study showed that there was an increase in the surface roughness of the composite resin after immersion in black tea for 1, 2, 3, and $4 \mathrm{~h}$. Statistical 
analyses via the paired t-test and one-way ANOVA also showed a significant difference. This indicates that the immersion of the nanofiller composite resin in black tea can increase the surface roughness of the resin.

One of the characteristics of the composite resin is its solubility in a solution. The solubility of a composite resin surface particle can be one of the causes for the decreased wear resistance causing erosion from the surface of the composite resin. In addition, another characteristic, i.e., water sorption, can also decrease the using time of the composite resin by increasing the resin component, hydrolyzing silane, and causing a little crack gap that can increase the probability of staining in the restoration $[5,6]$.

Consumption of certain types of drinks such as coffee, tea, and soft drinks can affect the esthetic and physical characteristics of the composite resin, which in turn can affect the quality of restoration. Black tea is one of the most consumed acidic drinks in Indonesia [2]. It has been reported that an acidic drink can affect the integrity of the composite resin surface by softening the matrix and causing a loss of the structural ions such as aluminum and silicon from the glass phase, thus affecting the durability of the material [6]. In an acidic condition, tooth restoration material degrades over time. Surface degradation of this composite resin has been linked with the component, the distribution of the filler particle, and the composition of the matrix resin [7].

The results of this study are also consistent with some of the results of previous studies. Cresus et al (2011) demonstrated an increase in the composite resin surface roughness after immersion in acidic drinks for 15 days [3]. Reddy et al (2013) also demonstrated an increase in the composite resin surface roughness after immersion in three most consumed drinks, including tea, coffee, and cola, for 30 days [8]. The present study also demonstrated an increase in the surface roughness of the nanofiller composite resin after immersion in black tea for 1, 2, 3, and $4 \mathrm{~h}$. The surface roughness values obtained in the immersion groups are still clinically acceptable because they are still $\leqslant 0.2 \mu \mathrm{m}$, as has been stated by Schmitt et al (2011) [4].

Surface roughness of a restoration material can lead to the growth of microorganisms in the oral cavity, and the food remaining in the oral cavity can also trigger the formation of plaque. The increase in surface roughness of the restoration material can increase the adherence of plaque and harm the teeth and the periodontal tissues, thereby causing discoloration of the surface of the material and fatigue failure [1].

\section{Conclusion}

The results of this study showed that there was a significant difference in the surface roughness of the nanofiller composite resin after immersion in black tea for 1, 2, 3, and $4 \mathrm{~h}$.

\section{References}

1. Ganulol N, Yilmaz F. The effects of finishing and polishing technique on surface roughness and color stability of nanocomposites. J Dent. 2012; 4: 64-70.

2. Sharangi AB, Siddiqui MDW, Aviria JED. Black Tea Magic: Overview of Global Research on Human Health and Therapeutic Potentialities. J Tea Sci Res 2014; 4 (1):1-16 
in Hamoud I M, Eklader H A, Badawi M F. Microloeakage of Nanofilled Composite Resin Restorative Material. J Biomat Nanobiotechnol 2011; 2: 320-34.

3. Cresus V, Gouvea D, Bedran LM, Fasria MA, Ferreira NC. Surface Roughness and Translucency of Resin Composites after Immersion in Coffee and Soft Drink. Acta Odontal 2011; 24(1): 3-7

4. Schmitt VL, Rontani RMP, Naufel FS, Nahsan FPS, Sinhoretti MAC, Baseggio W. Effect of Polishing Procedures on Color Stability and Surface Roughness of Composite Resin. ISRN Dentistry 2011; 1-6.

5. Lee SY, Kim HC, Hur B, Park JK. Surface Roughness and Color Stability of Various Composite Resin. J Korean Academy Conser Dent 2007; 32(6): 542-9.

6. Erdemir U, Yildiz E, Eren ME. Effects of Sports Drink on Color Stability of Nanofilled and Microhybrid Composites after Long-Term Immersion. J. Dent. 2012; 4: 55-63.

7. Poggio C, Dagna A, Chiesa M, Colombo M, Scribante A. Surface Roughness of Flowable Resin Composites Eroded by Acidic and Alcoholic Drinks. J Conser Dent 2012; 15(2): 137-40.

8. Reddy PS, Tesjaswi KLS, Shetty S et al. Effects of Commonly Consumed Beverages on Surface Roughness and Color Stability of the Nano, Microhybrid and Hybrid Composite Resins: An in vitro Study. J Contemp Dent Pract 2013; 14(4): 718-23. 